\title{
Synergistic science with Euclid and SKA: the nature and history of Star Formation
}

\author{
Paolo Ciliegi and Sandro Bardelli \\ INAF - Osservatorio Astronomico di Bologna, Via Ranzani 1, 40127 Bologna, Italy \\ E-mail: paolo.ciliegi@oabo.inaf.it
}

\begin{abstract}
We explored the impact of the synergy between the Euclid near-infrared photometric surveys and the SKA radio continuum surveys on the studies of the cosmic star formation. The Euclid satellite is expected to perform a Wide and Deep photometric surveys to an infrared limit of $\mathrm{H} \simeq 24$ and $\mathrm{H} \simeq 26$ respectively and a spectroscopy survey with a flux limit of $\sim 3 \times 10^{-16} \mathrm{erg} \mathrm{cm}^{-2} \mathrm{~s}^{-1}$ in the $\mathrm{H}_{\alpha}$ line. Combining the $\mathrm{H}$ band Euclid selected samples with the ground based ancillary data (fundamental for the SFR estimation) we will be able to detect the star forming galaxies down to SFRs of order of unit to $\mathrm{z} \sim 2$ and down to $\mathrm{SFR} \simeq 10$ to $\mathrm{z} \sim 3$, sampling the majority of the star forming galaxies up to $\mathrm{Z} \sim 3$ and beyond and placing definitive constraints on the star formation history of the universe at $\mathrm{z}<4-5$ (is there a peak a $\mathrm{z} \sim 2$ or a plateau at $1 \lesssim \mathrm{z} \lesssim 5$ ?) and on the galaxies evolution models. The only tools able to provide a accurate dust-free calculation of their SFR are the SKA continuum surveys.

The observational parameters of the Deep Tier SKA1 reference survey (a $0.2^{\prime \prime}-0.5^{\prime \prime}$ resolution and a $5 \sigma$ detection limit of $1 \mu \mathrm{Jy}$ over $30 \mathrm{deg}^{2}$ at Band 1/2) are the perfect complement of the Euclid survey. We showed, in fact, that with this kind of SKA survey we will be able to determine a dust unbiased SFR for a huge fraction $(\sim 85 \%)$ of the Euclid SFG providing strong constraints on the star formation history of the Universe. Moreover, the high angular resolution will provide an important tool to study the star formation history not only without dust contamination but also without AGN contamination. Finally, we suggest that during the SKA2 configuration a similar survey must be conducted also at higher frequency $(\sim 10 \mathrm{GHz})$ in order to allow the identification and separation of thermal and non-thermal radio emission components in higher redshift star forming galaxies
\end{abstract}

Advancing Astrophysics with the Square Kilometre Array

June 8-13, 2014

Giardini Naxos, Italy 


\section{Introduction}

Understanding how galaxies form and the physical processes that drive their evolution has been an active field of study ever since galaxies were observationally established as objects external to our Milk Way. Modern galaxy formation theory is developed within the cold dark matter plus dark energy scenario where galaxies form when gas condenses at the centre of dark matter halos, following the radiative cooling of baryons. This leads to the formation of neutral hydrogen clouds in which denser regions can cool further and form molecular clouds where, in turn, star formation takes place (see Bough 2006 and Benson 2010 for recent review on galaxy formation theory). However, how and when galaxies build up their stellar mass is still a major question in observational cosmology. While a general consensus has been reached in the last few years on the evolution of the galaxy stellar mass function (Dickinson et al. 2003, Fontana et al. 2006) the evolution of the star formation rate (SFR) as function of redshift and stellar mass $\operatorname{SFR}(M, z)$ still remains unclear.

Initial determinations of the evolution of the SFR in the universe were based on optical observations which reveal rest-frame ultraviolet luminosities of starbursts at high redshift (Madau et al. 1996, Lilly et al. 1996). Uncertain corrections for extinction are the greatest limitation of such studies, and the importance of dust obscuration has become increasingly emphasized by several factors : the dependence of obscuration on galaxy mass (more massive galaxies are more obscured) and on SFR (higher SFR means more obscuration) (Hopkins et al. 2001, Garn and Best 2010) and the discovery of a large population of starburst galaxies among the dusty sources found by the Spitzer Space Telescope (Dey et al. 2008). After about two decades of studies, it has now became clear that we cannot understand galaxy evolution and SFR without accounting for the energy absorbed by dust and re-emitted in the far-infrared (FIR) and sub-millimetre regions. More than 15 years ago using the SCUBA sub millimetre data, Hughes et al. $(1998,2002)$ found a star formation rate density (SFRD) that increases steeply to $\mathrm{z} \sim 1$, then flattens between $\mathrm{z} \sim 1$ and $\mathrm{z} \sim 3$ and decreases at $\mathrm{z} \gtrsim 3$. A very similar trend has been recently confirmed by Gruppioni et al. (2013) using the FIR data from the Herschel satellite. However, although an extinction-free measure, the interpretation of FIR and sub-millimetre emission is complex. Variations in the dust composition, content, temperatures and distribution along the line of sight affect the fraction of UV photon absorbed, while a portion of the FIR emission could arises from dust heated by older stars (Bendo et al. 2010, Li et al. 2010).

An independent estimate of the SFR in a galaxy, not biased by the galaxy's dust content, is provided by its radio continuum emission. This is due to processes such as the free-free emission from HII regions and the synchrotron radiation from relativistic electron from supernova (SN) remnants (Condon 1992). Over the last few years, several studies based on the radio emission (Seymour et al. 2008, Pannella et al. 2009, Karim et al. 2011) confirmed the rapid increase of the star formation rate density (SFRD) from $z=0$ to $z \sim 1$ in very good agreement with the results already obtained at other wavelengths, while at redshift greater than $z \sim 1.5$ there is still a significant discrepancy between the SFRD estimated from different wavelengths. These discrepancies do not allow us to establish unambiguously the presence of a peak in the SFRD at $\mathrm{z} \sim 2$ or a plateau at $\mathrm{z} \gtrsim 1.5$, severely limiting our conclusions on galaxies evolution models. These results are summarized in Fig. 1 from Ilbert et al. (2013) where the SFRD inferred from different wavelengths is plotted against the redshift. 


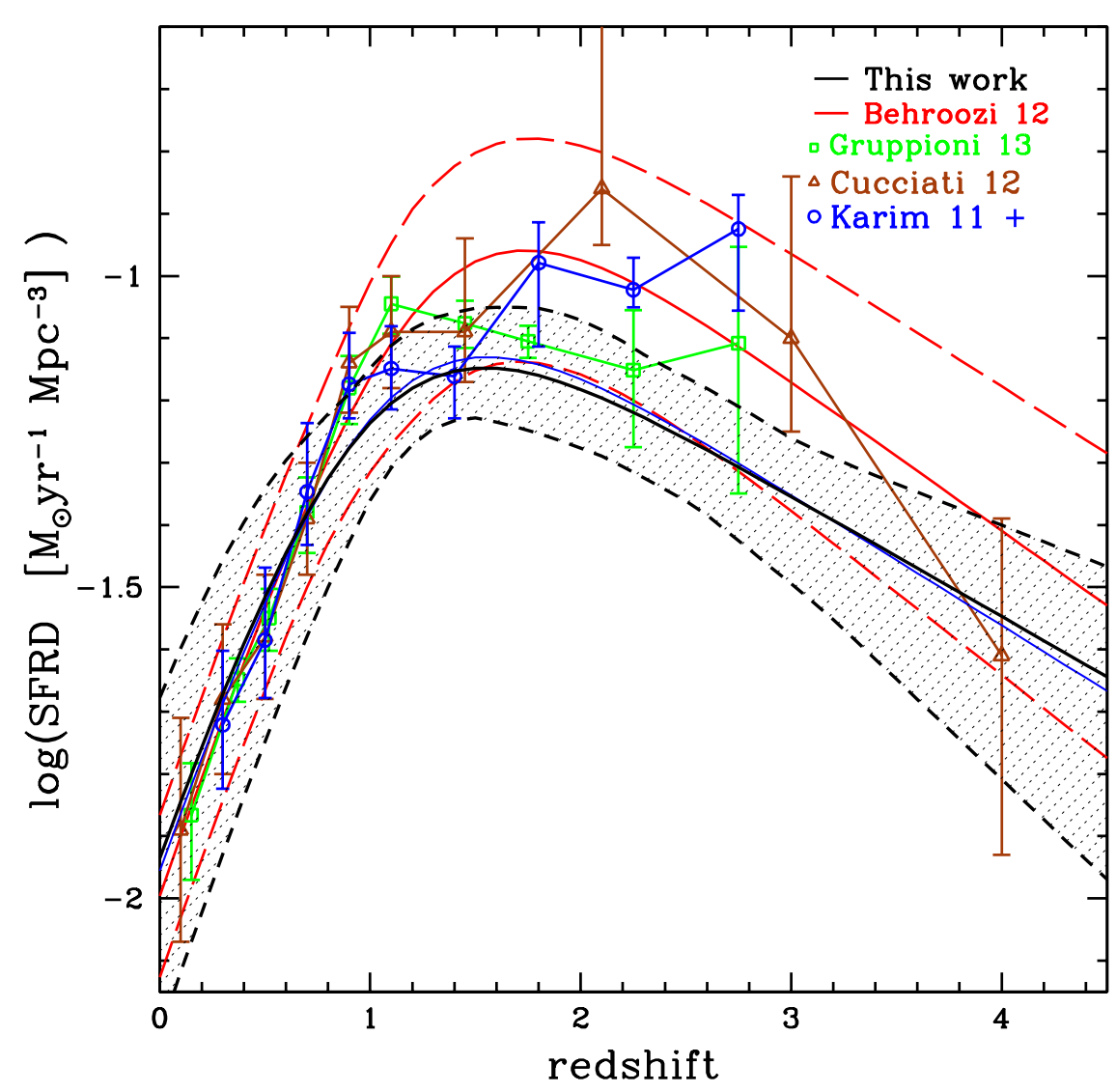

Figure 1: Star formation rate density inferred from different wavelengths (figure from Ilbert et al. 2013) including: direct measurements compiled by Behroozi et al. (2013) (red solid line with dashed lines for associate uncertainties), SFRD derived from the UV and IR luminosity function from Cucciati et al. (2012), and Gruppioni et al. (2013) (brown triangles and green squares, respectively), radio estimates from Karim et al. (2011) (blue open circle) and finally the SED fitting SFRD derived from the K-band selected UltraVISTA sample from Ilbert et al. (2013) (black solid line and dashed area corresponding to $1 \sigma$ errors).

However, while the obvious advantage of the radio emission as a tracer for star formation is its independence on any correction for dust attenuation, there are two major drawbacks : i) the general low sensitivity to the normal galaxy population even in the deepest radio surveys to date which usually limits the analysis at high redshift to a stacking approach ; ii) as for the UV and far infrared wavelength, radio emission is not only produced by star formation but also by active galactic nuclei (AGNs) and therefore we need to separate the radio sources into those whose radio emission is AGN dominated and those that are consistent with being dominated by star formation (Seymour et al. 2008, Smolcic et al. 2008, Bardelli et al. 2010). To overtake these problems a new generation of multi-wavelengths surveys (in terms of sensitivity, resolution and area covered) are needed in order to detect the faintest radio sources and to uniquely characterize their physical properties (origin of radio emission, redshift, mass). The next two decades will open a new astronomical era to address the open questions discussed above. Several new generation facilities are, in fact, planned to be 
realised and to work at different wavelengths. In the radio regime the advent of the revolutionary interferometer SKA will be preceded by a number of next-generation radio telescope and upgrade, including APERTIF, eMERLIN, JVLA, LOFAR and the two SKA precursors: ASKAP (Australia) and MeerKAT (South Africa). All these facilities are part of a wider context of new generation instruments, from the optical and near infrared band (LSST, E-ELT, TMT, GMT) to the X-ray band (eRosita, Athena), whose data will be able to provide a new panchromatic view of the Universe. However, the huge amount of data that will be produced by all these facilities forces to establish strong synergies in order to maximize the use of data.

For a more comprehensive discussion on the potential role played by the SKA in addressing the history of star formation we refer to Jarvis et al. (2015, this Volume). In this contribution we concentrate on how a synergy between the SKA continuum survey and the Euclid data (combined with the optical ancillary data for the SFR determination) will revolutionise our current knowledge on the nature and history of the star formation history, while for a description of the cosmological implication of the SKA-Euclid synergy see Kitching et al. (2015, this Volume).

\section{The Euclid mission}

Euclid (PI Y. Mellier) is a Medium Class mission selected by ESA within the Cosmic Vision programme and is aimed to "Understand the nature of Dark Energy and Dark Matter" using several cosmological probes. The launch is scheduled for 2020 and the end of the nominal mission is foreseen for late 2026.

For details of the mission, scientific goals and organization see the Euclid Red Book (Laureijs et al. 2011). The payload comprises two wide field instruments: a visible (VIS) and a near infrared photometric and spectroscopic instrument (NISP P and NISP S). The visible channel is aimed for weak lensing and will have a plate scale of 0.10 arcsec in a wide red band $(\mathrm{R}+\mathrm{I}+\mathrm{Z}, 0.55$ to 0.92 $\mu \mathrm{m})$. The NIR instrument in the photometric mode provides deep photometric data in three NIR bands ( $\mathrm{Y}, \mathrm{J}$ and $\mathrm{H}$ ). The spectroscopic mode operates in the 1.0-2.0 $\mu \mathrm{m}$ range and provides slitless spectra at a spectral resolution of $R \sim 250$.

Euclid is expected to perform a Wide Survey of $\sim 15,000 \mathrm{deg}^{2}$ to an infrared limit of mag=24 (Y,J,H at $5 \sigma$ detection for a point source) and to a visible limit of 24.5 (at $10 \sigma$ for extended sources). During this survey, the spectroscopy will have a flux limit of $\sim 3 \times 10^{-16} \mathrm{erg} \mathrm{cm}^{-2} \mathrm{~s}^{-1}$ (at $3.5 \sigma$ ) in the $\mathrm{H}_{\alpha}$ line. A Deep Survey will be done on a $40 \mathrm{deg}^{2}$ area and will reach a limit $\sim 2$ magnitude deeper in the NIR bands. It is expected that Euclid will deliver a dataset with images and photometry of more than a billion galaxies and several million of spectra.

Finally, in order to achieve the photometric redshift error of $\sigma[(1+z) / z] \sim 0.04$ required from the cosmological studies and a reliable SED fitting and SFR, the Euclid photometric data in the Y, $\mathrm{J}$ and $\mathrm{H}$ bands will be combined with deep ground based optical data. During the Euclid mission several new optical ground - based instruments optimized for deep, large area photometric surveys will be available, both in the north (CFHT, Pan-STARRS, SUBARU/HSC, LSST-South) and south (Blanco $4 \mathrm{~m} / \mathrm{DES}$ ) hemisphere. The combination of EUCLID and ground based data will provide a unique baseline of optical counterparts to study the star formation history of the universe with the SKA data, although we should keep in mind that a NIR (Euclid) selected sample of galaxies could be biased in favour of relatively massive galaxies at high redshift. 


\section{The Star Formation History}

The cosmic star formation history has been studied thoroughly over the past decade and half. As shown in Fig. 1 the space density of the star formation rate declines by an order of magnitude between a redshift of unit and zero, while at higher redshift the SFRD is almost flat when estimated al longer wavelengths (far infrared and radio) and shows a decline when estimated at shorter wavelengths (optical and near-infrared). Since at redshift $1 \leq z \leq 3$ the star formation activity appears to be dominated by dusty, heavily obscured, star forming galaxies (Caputi et al. 2007, Murphy et al. 2011a), much of the discrepancy among these estimates could arise by a poor determination of the dust extinction in the optical near-infrared band and how SFRs in different galaxy populations are determined. Thus, deep radio continuum surveys can provide an important tool for measuring the cosmic star formation history of the Universe out to $z \sim 5$, down to very modest SFRs. Moreover, because star-forming galaxies are discovered in various way, it is vital to compare various methods for measuring the SFR in galaxies.

In the next decade, facilities of new generation in the optical near infrared band (Euclid) and radio band (SKA) will give us a unique opportunity to open a new window on our knowledge of the star formation history of the Universe. In particular the availability of deep SKA radio continuum data and Euclid deep near infrared data (with its multi wavelengths ancillary data) will give us the opportunity to study with unprecedented accuracy and precision the following issues :

- The Star Formation History out to $z \sim 5$, down to very modest SFRs. As recently shown by Rodighiero et al. 2011, starburst galaxies (defined as sources with SFR $\gtrsim 100-1000 \mathrm{M}_{\odot} / \mathrm{yr}$ in the mass range $10^{10}-10^{11} \mathrm{M}_{\odot}$ ), i.e. sources that have a SFR - stellar mass ratio greater than 4 times the main sequence (MS) for star-forming galaxies defined by Daddi et al. (2007), represent only $2 \%$ of mass-selected star-forming galaxies and account for only $10 \%$ of the cosmic SFR density at the cosmic peak of the star formation activity ( $\mathrm{z} \sim 2)$ (Rodighiero et al. 2011). The bulk of the star formation history is driven by "normal" star forming galaxies (SFG) that have SFR $\sim 10-20 \mathrm{M}_{\odot} / \mathrm{yr}$ at a stellar mass $\sim 10^{10} \mathrm{M}_{\odot}$ and SFR $\sim 100$ only for galaxies with stellar mass of $\sim 10^{11} \mathrm{M}_{\odot}$.

Therefore, in order to have a direct, dusty unbiased measure of the bulk of the SFG responsible of SFR history of the Universe, a new generation of extremely deep continuum radio data are needed. In fact, as shown by Seymour et al. 2008 the actual generation of radio surveys are able to sample only the highest SFR objects at any given redshift. A radio survey with a $4 \sigma$ detection limit of $30 \mu \mathrm{Jy}$ is able to detect star forming galaxies with a SFR of $\sim 10-20$ $\mathrm{M}_{\odot} / \mathrm{yr}$ only locally $\left(\mathrm{z} \lesssim 0.5\right.$ ), while at $\mathrm{z} \gtrsim 1$ only starburst galaxies with $\mathrm{SFR} \gtrsim 100 \mathrm{M}_{\odot} / \mathrm{yr}$ are detected: the bulk of the SFG responsible of the Star Formation History of the Universe is completely missed. This is well represented in Fig. 2 from Seymour et al. (2008), where the SFR is plotted against the redshift for different stellar masses ranges.

The photometric deep survey from the Euclid satellite will be able to detect normal star forming galaxies with low SFR (few $\mathrm{M}_{\odot} / \mathrm{yr}$ ) out to $\mathrm{z} \sim 3-4$ (see next section). However, considering that a new generation of FIR facilities are not scheduled so far, an accurate dustunbiased estimate of the SFR of the SFG can be obtained only with deep radio surveys. The SKA deep continuum radio surveys are the perfect tool to achieve this goal. In the 


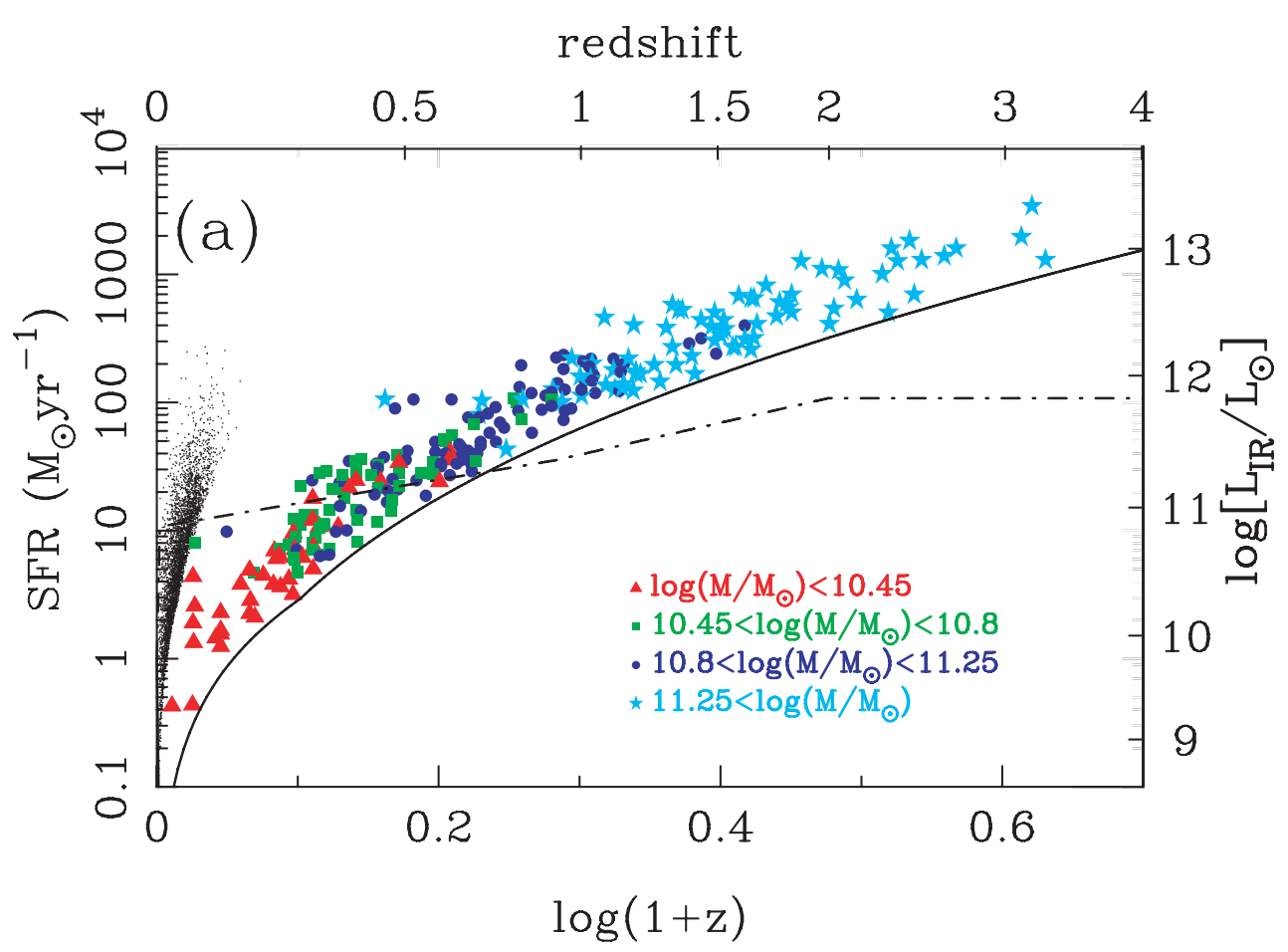

Figure 2: Star formation rate as function of redshift from the $13^{H}$ XMM/Chandra Dee Field (figure from Seymour et al. 2008), one of the deepest radio survey actually available with a $4 \sigma$ detection limit of $30 \mu \mathrm{Jy}$. The different symbols represent different stellar masses ranges as indicated in the panel. The solid line represents the $1.4 \mathrm{GHz}$ detection limit and the dot dashed line indicates the 10 times $\mathrm{L}_{\star}$ of the radio star forming LF. The black dots are the SFGs from the local 6dF-NVSS sample. See Seymour et al. 2008 for more details.

next section we will consider different SKA surveys (in terms of depth, resolution and area covered) and we will investigate their impact (coupled with the Euclid data) on the study of the star formation history of the Universe.

\section{- Star Formation vs AGN activity in Radio Sources}

In addition to the classification of the sources based on the Euclid plus ground based multi wavelength data (via SED fitting, Smolcic et al. 2008, Bardelli et al. 2010, see also McAlpine et al. 2015, this Volume), the availability of deep radio and NIR infrared surveys gives us the opportunity to test at very faint flux levels (both in the NIR and radio bands) the methods to separate star forming from AGN activity using the radio to NIR flux ratio used by Seymour et al. (2008) down to a radio flux level of $30 \mu \mathrm{Jy}$.

In addition, the excellent high angular resolution of the SKA1 (from $\sim 0.2^{\prime \prime}$ to $\sim 1^{\prime \prime}$ ), corresponding to spatial resolution spanning sub-kpc to $\sim 10 \mathrm{kpc}$ at $\mathrm{z}>1$, will provide a unique opportunity to separate the core radio emission, likely associated to AGN activity, from more extended emission, likely associate to star forming regions, giving us the opportunity to study the star formation history not only without dust contamination but also without AGN con- 
tamination.

\section{- The nature of radio emission in star-forming galaxies}

Radio continuum emission from galaxies typically arises from two processes that are both tied to the SFR. At low frequency $(\leq 2 \mathrm{GHz})$, the radio continuum is dominated by nonthermal synchrotron emission arising from cosmic-ray electrons that have been accelerated by SN remnants and are propagating through the galaxy's magnetised interstellar medium. This physical link to massive star formation provides the foundation for the far infrared (FIR) - radio correlation. At high frequency $(\sim 10-100 \mathrm{GHz})$ the radio emission is dominated by thermal (free-free) radiation, which is directly proportional to the ionising photon rate of young, massive stars. However, while for the non-thermal radiation it is unclear how presumably unrelated physical processes (propagation of cosmic - ray electrons, magnetic field strength/structure, heating size and composition of dust grain) could conspire together to keep the FIR-radio relation intact, the free-free emission is largely extinction free and can be directly related to the star formation. Thus sensitive observations at radio frequency $\gtrsim 10 \mathrm{GHz}$, along with parallel deep observations at $1.4 \mathrm{GHz}$, will allow the identification and separation of thermal and non-thermal radio emission components in higher redshift star forming galaxies (Murphy et al. 2011b). The SKA1-MID instrument with its sensitivity up to $\sim 14 \mathrm{GHz}$ (Dewdney et al. 2013) is the perfect instrument to perform such observations.

\section{The Euclid and SKA synergetic view of the star formation history}

\subsection{The SKA surveys}

In this section we will investigate the impact of different SKA continuum surveys (in terms of depth, resolution and area covered) on the study of the SFR as a function of redshift in combination with the Euclid data. As reference SKA1 continuum survey we used the three-tiered survey at Band 1/2 reported in Seymor and Prandoni (2014) : 1) a Wide Tier 1000-5000 deg ${ }^{2}$ survey with $0.5^{\prime \prime}$ resolution with a $5 \sigma$ detection limit of $5 \mu \mathrm{Jy} / \mathrm{beam}$; 2) a Deep Tier 10-30 $\mathrm{deg}^{2}$ survey with $0.5^{\prime \prime}$ resolution with a $5 \sigma$ detection limit of $1 \mu \mathrm{Jy} / \mathrm{beam}$ and 3) an Ultra-Deep Tier survey of $1 \mathrm{deg}^{2}$ with $0.5^{\prime \prime}$ resolution with a $5 \sigma$ detection limit of $0.25 \mu \mathrm{Jy} / \mathrm{beam}$.

\subsection{The Euclid survey}

The Euclid wide survey down to a photometric limit 24 mag in the NIR band (see Section 2) has been assumed as our reference NIR survey. In order to simulate a reliable Euclid wide survey sample, we started from the deep photometric sample (version 1.8, Ilbert et al. 2009) available in the COSMOS field Scoville et al. (2007). This sample is a compilation of photometric data in the optical and NIR bands, covers an area of about $1.4 \mathrm{deg}^{2}$ and is limited to the optical magnitude $i \sim 28$ with completeness limit at $i \sim 26.5$. In Ilbert et al. (2009) all the intrinsic properties of the sources (like photometric redshift, star formation rate, mass) have been estimated through a Spectral Energy Distribution (SED) fitting procedure using 30 broad, intermediate and narrow bands from UV to mid-IR frequencies (see their Table 1). With the same catalogue and SED fitting procedure, we obtained an Euclid simulated sample in the $\mathrm{J}_{A B}$ and $\mathrm{H}_{A B}$ bands down to a magnitude limit of $\mathrm{H}_{A B}=24$ (Ilbert, private communication). 


\subsection{The SFR with Euclid and SKA}

In this section we test the capability of the simulated sample described above (ground based multi wavelength optical data down to $i \sim 28$ plus the Euclid simulated wide survey down to $\mathrm{H}_{A B}=24$ ) to observe the bulk of the star forming galaxies up to $\mathrm{z} \sim 3$. In Fig. 3 we plot the SFR obtained from SED fitting procedure (Ilbert et al. 2009) using all the available optical to NIR bands as a function of redshift: red dots show the SFR for the subsample with $\mathrm{H}_{A B}<24$ (detectable in the Euclid wide survey) while the black dots show the entire photometric sample limited to $i \sim 28$ without limitation in the $\mathrm{H}$ band. As shown in the figure, the $\mathrm{H}_{A B}<24$ limited sample ("Euclid sample") at a given redshift samples the region of higher SFR with respect the entire sample. This is due to the fact that with a NIR limited sample we favour the detection of relatively massive galaxies, losing low mass starburst galaxies. However, even though these mass and SFR limitations, the Euclid wide survey down to $\mathrm{H}_{A B}=24$ (combined with the ground based ancillary data for the determination of the SFR values), will allow us to detect the SFG down to SFRs of order of unit to $\mathrm{z} \sim 2$ and down to $\mathrm{SFR} \simeq 10$ to $\mathrm{z} \sim 3$. Better results, of course, will be obtained using a sample selected from the Euclid deep survey that will reach a limit $\sim 2$ magnitude deeper in the NIR bands over an area of $\sim 40 \mathrm{deg}^{2}$, combined with the ground based data for the SFR calculation. With these samples in our hands we are going to sample the majority of the massive star forming galaxies up to $\mathrm{z} \sim 3$ and beyond, placing definitive constraints on the star formation history of the universe at $\mathrm{z}<4-5$ (is there a peak a $\mathrm{z} \sim 2$ or a plateau at $1 \lesssim \mathrm{z} \lesssim 5$ ?) and on the galaxies evolution models.

However, while the Euclid data and its ancillary multi-wavelengths data will give us the opportunity to obtain informations on the intrinsic properties of the sources, including the SFR through the SED fitting procedure, an independent, dusty unbiased, estimation of the SFR of these sources is a fundamental step that must be obtained in order to ensure a clear picture of the cosmic star formation. Moreover a very deep radio continuum survey will ensure also the detection of the low mass starburst galaxies population that we could miss in a sample selected in the NIR band.

Considering the extremely low SFR of these sources (see Fig. 3) we have only one tool able to reach this scope : the SKA radio continuum survey. Following Bell (2003) we converted the radio flux detection limit of the four SKA surveys considered (see above) in SFR as function of the redshift, and we plot the relative curves in Fig. 3.

At a $5 \sigma$ detection limit of $5 \mu \mathrm{Jy}$ (Wide Tier SKA1 survey, solid blue line in Fig. 3) we are able to detect only a small part of the Euclid objects, from $\sim 25 \%$ at $z<1.4$ to $\sim 10 \%$ for higher redshifts, sampling only the region of the high SFR (the starburst region) and completely missing the sources (star forming galaxies) responsible of the bulk of the cosmic star formation (see Rodighiero et al. 2011). Therefore a radio survey at this flux level, similarly to the deepest radio survey actually available (see Fig. 2 and the black dashed line in Fig. 3 corresponding to the limit of the COSMOS radio survey of $50 \mu \mathrm{Jy}, 5 \sigma$ detection limit, Schinnerer et al. 2007) is not suitable to strongly constrain the galaxies evolutionary models, except through more uncertain statistical studies as the stacking analysis (see Pannella et al. 2009, Karim et al. 2011).

The $1 \mu \mathrm{Jy} 5 \sigma$ detection limit survey (Deep Tier) perform much better, detecting $\sim 85 \%$ of the Euclid objects in the redshift range considered, (white long dashed line in Fig. 3) and sampling the region of the star forming galaxies with low SFR (few $\mathrm{M}_{\odot} / \mathrm{yr}$ ) responsible of the bulk of the cosmic star formation. The Ultra Deep Tier survey, corresponding to a $5 \sigma$ detection limit of 0.25 
$\mu \mathrm{Jy}$ is plotted in Fig. 3 as solid green line. At this flux limit we detect almost all the star forming Euclid objects.

Finally, as reference, in Fig. 3 we show also the line (dot dashed yellow) corresponding to the spectroscopic Euclid sample, where the $\mathrm{H}_{\alpha}$ limit of $3 \times 10^{-16} \mathrm{erg} \mathrm{cm}^{-2} \mathrm{~s}^{-1}$ is converted in star formation rate following the formula of Moustakas et al. (2006). This sample corresponds roughly to the radio flux limit of $10 \mu \mathrm{Jy}$, with the limitation in the derivation of the star formation history of the Universe discussed above. The limit in the SFR values sampled by the Euclid spectroscopic redshift strengthen the need for a strong synergy between Euclid and SKA, the latter being the only instrument able to provide a direct measure of the SFR for all the star forming galaxies (even with few $\mathrm{M}_{\odot} / \mathrm{yr}$ up to $\mathrm{z} \simeq 3-4$ ) that will be detected in the Euclid photometric surveys.

The results presented in this section are summarised in Table 1, where for each survey considered, we report the $5 \sigma$ detection flux limit $\left(\mu \mathrm{Jy} /\right.$ beam), the area covered (in $\mathrm{deg}^{2}$ ), an estimation of the total integration time needed to complete the survey with SKA, the value of the SFR sampled at $\mathrm{z} \simeq 1$ and $\mathrm{z} \simeq 2-3$ and the fraction of the Euclid SFGs that will be detected in the radio regime by the survey. Moreover, for comparison, we report also the values for the deepest already available surveys (VLA-COSMOS (Schinnerer et al. 2007) and 13 ${ }^{H}$ XMM/Chandra Deep Field (Seymour et al. 2008)), for the ongoing surveys that will be obtained with the actual upgraded instrumentation (JVLA COSMOS 3GHz Large Project, PI V. Smolčić) and for the SKA precursors ASKAP-EMU (Norris et al. 2011), APERTIF-WODAN and MeerKAT-MIGHTEE. Finally, for the Deep Tier survey we considered also the values that can be reached during an "early-science" phase of deployment for each SKA1 component, where sensitivity has grown to about $50 \%$ of its fully specified level, and during the final SKA phase (SKA2), when the sensitivity, resolution and field of view will be improved by a factor 10-20 in comparison to SKA1.

\section{Conclusion}

The radio continuum surveys have been largely used over the past decade to study the star formation history of the Universe using their capacity to penetrate significant dust obscuration. However, even at the deepest radio flux limit of the available surveys, only the sources with the highest star forming rate ( $\left.\gtrsim 100-200 \mathrm{M}_{\odot} / \mathrm{yr}\right)$ are detected at redshift greater than $\mathrm{z} \sim 1$ and the bulk of the normal star forming galaxies responsible for $\sim 90 \%$ of the cosmic SFR density at $\mathrm{z} \sim 2$ are completely missed.

In this paper we showed how a strong synergy between two revolutionary facilities (Euclid and SKA), which will become operative during the next decade and the NIR and radio bands, will open a new window in our knowledge on the galaxies evolutionary models. In particular we showed that combining the $\mathrm{H}$ band Euclid selected samples with the ground based ancillary data (fundamental for the SFR calculation) we will be able to detect the majority of the SFG responsible of the cosmic SFR density and that the only tools able to provide an accurate dust-free calculation of their SFR are the SKA continuum surveys. Moreover a very deep radio continuum survey will ensure also the detection of the low mass starburst galaxies population that we could miss in a NIR selected sample.

We considered the SKA1 Reference Survey in Band 1/2 reported in Seymour and Prandoni (2014) and we showed that with the Deep Tier Survey (obtainable with a reasonable amount of time, 
Table 1: Radio continuum survey and their SFR observability

\begin{tabular}{|c|c|c|c|c|c|c|}
\hline Survey & $\begin{array}{r}\text { Flux } \\
(\mu \mathrm{Jy})\end{array}$ & $\begin{array}{l}\text { Resolution } \\
(\operatorname{arcsec})\end{array}$ & $\begin{array}{c}\text { Area } \\
\left(\operatorname{deg}^{2}\right)\end{array}$ & $\begin{array}{l}\text { Time } \\
\text { SKA }\end{array}$ & $\begin{array}{r}\text { SFR sampled } \\
\left(\mathrm{M}_{\odot} / \mathrm{yr}\right) \\
\end{array}$ & $\begin{array}{c}\% \text { Euclid } \\
\text { SFG }\end{array}$ \\
\hline \multicolumn{7}{|c|}{ Already available surveys and SKA Precursors } \\
\hline $\begin{array}{l}\text { Deep Radio Survey } \\
\text { already available }\end{array}$ & 50 & $\gtrsim 1$ & $\begin{array}{c}\text { few } \\
(\sim 2-3)\end{array}$ & - & $\begin{array}{r}\gtrsim 20 \text { at } \mathrm{z} \simeq 1 \\
\gtrsim 200 \text { at } \mathrm{z} \simeq 2-3\end{array}$ & $1-2$ \\
\hline $\begin{array}{l}\text { EMU + WODAN } \\
\text { (SKA Precursors) }\end{array}$ & 50 & 10 & all sky & - & $\begin{array}{r}\gtrsim 20 \text { at } \mathrm{z} \simeq 1 \\
\gtrsim 200 \text { at } \mathrm{z} \simeq 2-3\end{array}$ & $1-2$ \\
\hline $\begin{array}{l}\text { Ongoing deep } \\
\text { survey (JVLA) }\end{array}$ & 10 & 0.65 & 2 & - & $\begin{array}{r}\gtrsim 10 \text { at } \mathrm{Z} \simeq 1 \\
\gtrsim 100 \text { at } \mathrm{z} \simeq 2-3\end{array}$ & $5-10$ \\
\hline $\begin{array}{l}\text { MeerKAT-MIGHTEE T2 } \\
\text { (SKA Precursors) }\end{array}$ & 5 & 3.5 & 35 & 1950 & $\begin{array}{r}\gtrsim 8 \text { at } \mathrm{z} \simeq 1 \\
\gtrsim 60 \text { at } \mathrm{z} \simeq 2-3\end{array}$ & $10-25$ \\
\hline $\begin{array}{l}\text { MeerKAT-MIGHTEE T3 } \\
\text { (SKA Precursors) }\end{array}$ & 0.5 & 3.5 & 1.0 & 1700 & $\begin{array}{r}\gtrsim 0.4 \text { at } \mathrm{z} \simeq 1 \\
\gtrsim 1-2 \text { at } \mathrm{z} \simeq 2-3\end{array}$ & 90 \\
\hline \multicolumn{7}{|c|}{ SKA1 reference surveys at Band 1/2 (Seymour and Prandoni 2014) } \\
\hline Wide Tier & 5 & 0.5 & 5000 & $\sim 1 \mathrm{yr}$ & $\begin{array}{r}\gtrsim 8 \text { at } \mathrm{z} \simeq 1 \\
\gtrsim 60 \text { at } \mathrm{z} \simeq 2-3\end{array}$ & $10-25$ \\
\hline Deep Tier & 1 & 0.5 & 30 & $\sim 2000 \mathrm{hrs}$ & $\begin{array}{r}\gtrsim 0.5 \text { at } \mathrm{z} \simeq 1 \\
\gtrsim 2-3 \text { at } \mathrm{z} \simeq 2-3\end{array}$ & 85 \\
\hline Ultra Deep Tier & 0.25 & 0.5 & 1 & $\sim 2000 \mathrm{hrs}$ & $\begin{array}{l}\gtrsim 0.2 \mathrm{z} \simeq 1 \\
\gtrsim 1 \mathrm{z} \simeq 2-3\end{array}$ & 100 \\
\hline \multicolumn{7}{|c|}{ SKA1 early science and SKA2 } \\
\hline $\begin{array}{l}\text { Early Deep Tier } \\
(50 \% \text { SKA } 1)\end{array}$ & 2 & 0.5 & 30 & $\sim 1000 \mathrm{hrs}$ & $\begin{array}{r}\gtrsim 1 \text { at } \mathrm{z} \simeq 1 \\
\gtrsim 10 \text { at } \mathrm{z} \simeq 2-3\end{array}$ & 50 \\
\hline $\begin{array}{l}\text { Deep } \\
\text { SKA }\end{array}$ & 0.1 & $\lesssim 0.1$ & 30 & $\sim 40 \mathrm{hrs}$ & $\begin{array}{r}\gtrsim 0.1 \mathrm{z} \simeq 1 \\
\gtrsim 0.5 \mathrm{z} \simeq 2-3\end{array}$ & 100 \\
\hline
\end{tabular}

$\sim 2000$ hrs) we will be able to determine a dust unbiased SFR for a huge fraction $(\sim 85 \%)$ of the SFG detected by Euclid providing strong constraints on the star formation history of the Universe. Moreover the angular resolution of $\sim 0.2-0.5$ arcsec will ensure an unambiguous identification of the radio sources and will provide an important tool to separate the core radio emission (likely associate to AGN activity) from the outer emission likely associate to star forming regions, giving us the opportunity to study the star formation history not only without dust contamination but also without AGN contamination.

Moreover we showed that during the early science phase, when the SKA1 sensitivity will be $\sim 50 \%$ of its fully specified level, we will be able to detect about $50 \%$ of the Euclid SFG (see Table 1), starting to shed a light on properties (in terms of SFR) of the galaxies responsible of the bulk of the cosmic star formation. Finally, during the SKA2 phase, when the sensitivity, resolution and field of view will be improved by a factor 10-20 in comparison to the SKA1 phase, this kind of radio survey could be easily obtained in less than 2 days. However, at that time it will be desirable to increase the area covered and, more important, to obtain an high frequency $(\sim 10 \mathrm{GHz})$ survey 


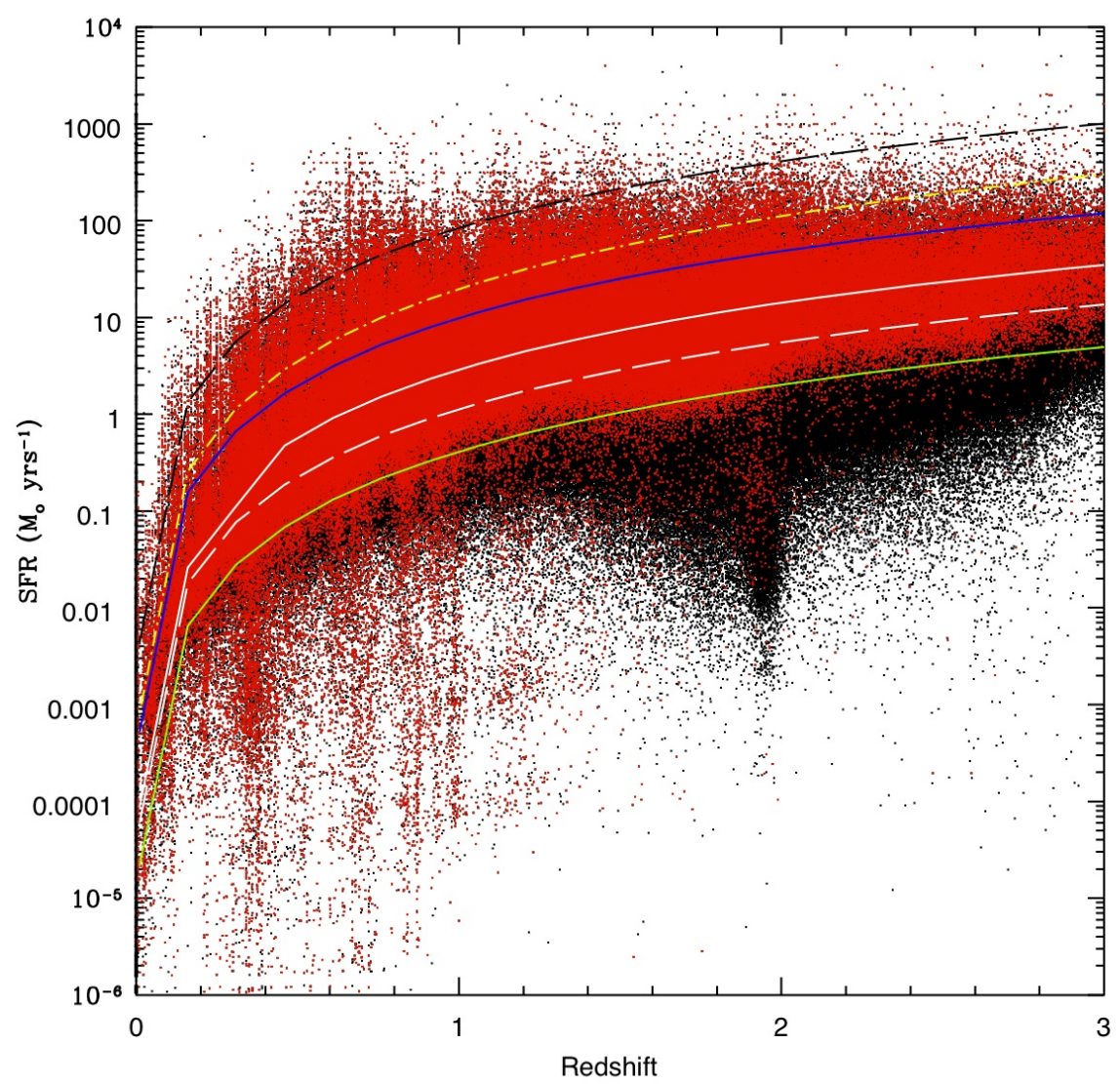

Figure 3: Star formation rate as function of redshift. SFR obtained from SED fitting procedure (Ilbert et al. 2009) for the simulated Euclid wide survey limited at $H<24$ are plotted as red dots, while the entire photometric sample limited to $i \sim 28$ with a completeness limit at $i \sim 26.5$ is plotted as black dots. The curves represents the SFR detection limit as function of redshift for the Euclid spectroscopic sample (dotdashed yellow line) and for different radio $5 \sigma$ detection flux limits : $50 \mu \mathrm{Jy}$ (dashed black line), $5 \mu \mathrm{Jy}$ (solid blue line), $2 \mu \mathrm{Jy}$ (solid white line), $1 \mu \mathrm{Jy}$ (dashed white line) and $0.25 \mu \mathrm{Jy}$ (solid green line)

with a similar depth, resolution and area covered in order to identify and to separate the thermal and non-thermal radio emission components in higher redshift star forming galaxies.

$0.0 \mathrm{pt}$

\section{REFERENCES}

Bardelli, S, et al, 2010, A\&A, 511,1

Behrooz,i P.S., Wechsler R.H. \& Conroy C., 2013, ApJ, 770, 57

Bell, E.F., 2003, ApJ, 586, 794

Bendo, G.J., et al., 2010, A\&A, 518,L65

Benson, A.J., 2010, Phys. Rep., 495, 33

Bough, C.M., 2006, Report on Progress in physics, 69, 3101 
Braun, R., 2014, "SKA1 Imaging Science Performance”, Document number SKA-TEL-SKO-DDXXX, Rev A, Draft 2

Caputi, K. I. , et al, 2007, ApJ, 660, 97

Condon J.J, 1992, ARAA, 30, 575

Cucciati O., et al., 2012, A\&A, 548, 108

Daddi, E., et al., 2007, ApJ, 670, 156

Dey, A. et al, 2008, ApJ, 677, 943

Dewdney, P., Turner, W.,Millenaar, R., et al., 2013, "SKA1 System Baseline Design", Document number SKA-TEL-SKO-DD-001 Revision 1

Dickinson, M., et al., 2003, ApJ, 587, 25

Fontana, A., et al., 2006, A\&A, 459, 745

Garn, T. and Best ,P.N., 2010, MNRAS, 409,421

Gruppioni, C., et al. , 2013, MNRAS, 436, 2875

Hopkins, A.M, et al., 2001, AJ, 122,288

Hughes, D.H. et al. 1998, Nature, 394, 241

Hughes, D.H. et al. 2002, MNRAS, 335, 871

Ilbert,. O., et al. , 2009, ApJ, 690, 1236

Ilbert, O. et al. 2013, A\&A, 556,55

Jarvis M. J., Bacon, D., Blake, D., et al., 2015, “Cosmology with SKA Radio Continuum Surveys”, in proc. Advancing Astrophysics with the Square Kilometre Array, PoS(AASKA14)018

Kitching T. D., Bacon, D., Brown, M. L., et al., 2015, “Euclid-SKA Synergies”, in proc. Advancing Astrophysics with the Square Kilometre Array, Pos(AASKA14)146

Karim, A., et al., 2011, ApJ, 2011, 730, 61

Laureijs, R. et al. , 2011', Euclid Definition Study Report, arXiv:1110.3193, [astro-ph.CO]

Li, Y., et al, 2010, ApJ, 725, 677

Lilly, S.J. et al., 1996, ApJL, 460, L1

McAlpine K., Prandoni, I., Jarvis, M., et al., 2015, "The SKA view of the Interplay between SF and AGN Activity, and its role in Galaxy Evolution”, in proc. Advancing Astrophysics with the Square Kilometre Array, PoS(AASKA14)083

Madau, P. et al., 1996, MNRAS, 283, 1388

Moustakas, J., Kennicutt R.C. \& Tremonti C.A., 2006, ApJ 642, 775

Murphy, E. J., et al., 2011a, ApJ, 732, 126

Murphy, E. J., et al., 2011b, ApJ, 737, 67

Norris, R. , et al, 2011, PASA, 28, 215

Pannella, M. , et al., 2009, ApJ, 698, L116

Rodighiero, G., et al., 2011, ApJL, 739, L40

Schinnerer, E., et al, 2007, ApJS, 172, 46

Scoville, N., et al., 2007, ApJS, 172, 1

Seymour, N., et al., 2008, MNRAS, 386, 1695

Seymour, N. and Prandoni I., 2014, Continuum Science Assessment Workshop Summary, SKABD-09-05c

Smolčić V., et al., 2008, ApJS, 177,14 